\section{Community care of individuals at risk of suicide: the Life Promotion Clinic model}

\author{
Kairi Kolves, Urska Arnautovska, \\ Angelo De Gioannis, Diego De Leo \\ Life Promotion Clinic, Australian Institute \\ for Suicide Research and Prevention, \\ Griffith University, Australia
}

\section{Abstract}

Assistance to suicidal patients is problematic both at the hospital and community care level. Inadequacy of facilities, pressured personnel, long waiting time, and professional and social stigmatization are just some of the many issues that interfere with successful treatment. The goal of this paper is to present the functioning of the Life Promotion Clinic (LPC), Australia, and describe its users. The LPC is the first specialized outpatient service in Australia dedicated to the treatment of individuals with suicidal thoughts and behaviors. A description of the service and characteristics of its clients (demographic, psychopathology, risk of suicide) are herein presented. Data were collected for 63 male and 175 female patients who attended the LPC over a threeyear period. Patients were mostly single females, aged up to 44 years, poorly educated, unemployed or on a pension/benefit. The majority of patients reported at least one suicide attempt, severe depression and anxiety scores, moderate-severe feelings of hopelessness, and high impulsiveness scores. Compared to females, male patients presented with more active desire to kill themselves and higher level of suicidal ideation. We can conclude that establishing a specialist service for treatment of individuals at increased risk for suicide requires consideration of both patient and clinicians needs. The LPC presents an innovative model of community service, capable of engaging patients with serious mental health issues, while making the service accessible to people from various social categories.

\section{Introduction}

Poorly perceived need for treatment and attitudinal/cultural barriers in help seeking, prevent many people, especially males, from receiving treatment after a suicide attempt. ${ }^{1}$ An analysis of 22 nationally representative samples worldwide, showed that less than half of people with suicidal behavior in the previous year had received some form of treatment. ${ }^{2}$ The receipt of treatment was especially low in middle- and low-income countries, where the perceived need for treatment was the most often reported reason for not seeking help. In contrast, in high-income countries the primary reasons for not seeking treatment were related to attitudinal barriers (e.g., trying to handle the problem on their own). In all countries, structural barriers, such as limited finances, lack of availability of treatment, transportation problems, and inconvenience of attending the treatment, were also reasons for not receiving care. ${ }^{2}$

A review of studies on attitudes towards clinical service underlined the importance of patient involvement in treatment, and also showed that many service users reported negative experiences with the discharge and referral to after-care services. ${ }^{3}$ These perceptions were supported by Lizardi and Stanley, ${ }^{4}$ who found that the transition from emergency to outpatient services is a crucial but often neglected step in treating suicidal people.

Difficulties with engaging in treatment are also likely to be complicated by high risk of repeated attempts after discharge. ${ }^{5}$ General hospital staff most often hold negative attitudes towards people who self-harm. ${ }^{6}$ These attitudes (often charged with feelings of irritation, anger, frustration, and helplessness) were found to be particularly expressed towards patients who repeatedly self-harmed a group at highest risk of subsequent suicide. 7,8

Notwithstanding these issues, the majority of people with suicidal ideation or behavior are willing to accept help in managing and minimizing their risk of suicide. ${ }^{3}$ Recognizing the need of suicidal individuals for specialist care, and considering that history of suicidal behavior is the most important predictor of subsequent self-harming behaviours, ${ }^{7}$ the Australian Institute for Suicide Research and Prevention (AISRAP) established the Life Promotion Clinic (LPC). The LPC was the first Australian community-based clinic specialized in assessing and treating individuals with suicidal ideation and behavior. The aims of this paper were to present the model of care of the LPC, and to describe its patients.

\section{Materials and Methods}

\section{The Life Promotion Clinic:} \section{background and development}

The rationale for establishing the LPC came from increased demand from community, government and non-government organizations for a specialized clinical service to provide comprehensive treatment to suicidal people (i.e., a service that is not routinely provided by
Correspondence: Diego De Leo, Life Promotion Clinic, Australian Institute for Suicide Research and Prevention, Griffith University, Messines Ridge Rd, Mt Gravatt, Queensland 4122, Australia.

Tel. +07.3735.1168 - Fax: +07.3735 .3450$

E-mail: d.deleo@griffith.edu.au

Key words: suicidal ideation and behavior, barriers to treatment, specialist outpatient service.

Acknowledgements: we would like to thank Griffith University for continuous support of the LPC, and hospital staff for entrusting the clinic with the care of their patients. Furthermore, we would like to acknowledge the work of all clinicians, Diane 0'Brien (mental health nurse), and Paulomi Doshi (clinic administrator), as well as Timothy Hall: all put substantial efforts in data collection, treatment, and engagement with patients of the LPC.

Contributions: the authors contributed equally.

Conflict of interests: the authors declare no potential conflict of interests.

Received for publication: 27 March 2013.

Revision received: 26 August 2013.

Accepted for publication: 1 September 2013.

This work is licensed under a Creative Commons Attribution NonCommercial 3.0 License (CC BYNC 3.0).

(C) Copyright K. Kolves et al., 2013

Licensee PAGEPress, Italy

Mental Illness 2013; 5:e12

doi:10.4081/mi.2013.e12

EDs, psychiatric inpatient settings or general hospital settings, or community mental health services).

This was supported by research showing that negative perceptions regarding hospital treatment and attitudes of hospital staff strongly influence help-seeking behavior. In fact, in a community survey performed in Queensland, $52.5 \%$ of people who have planned suicide, and $29.8 \%$ of people who have made a suicide attempt, did not seek professional help. ${ }^{9}$ Most of them refer to attitudes of hospital and community services personnel as reasons for not seeking help.

An additional motive for the implementation of the LPC was represented by the need for a treatment environment facilitating clinical research on protocols for suicidal people, able also to constitute a specialized training setting for psychiatry registrars and clinical psychologists.

On these grounds, AISRAP, in 2004, initiated the establishment of the LPC, by locating it in its main building at Mt Gravatt campus of Griffith University. The clinic employs consult- 
ant psychiatrists, psychiatry registrars, clinical psychologists, a mental health nurse, and administrative staff. Ordinarily, it is open to the public on Tuesdays-Wednesdays, 8.0018.00. The clinic is now accredited as a training facility of the Royal Australian and New Zealand College of Psychiatrists (RANZCP), and offers opportunities for both basic and advanced training (psychotherapy). The LPC also contributes to research, education and training of health professionals from outside Australia, as part of AISRAP's commitment as a WHO Collaborating Centre for Research and Training in Suicide Prevention. An agenda of educational seminars, open to the public, completes the programs of the LPC.

The clinic pursues the following objectives: i) to provide an appropriate outpatient service to people with suicidal ideation and behavior; ii) to collect data regarding treatment outcomes, so as to develop effective protocols; iii) to provide a setting for clinical training of psychiatry registrars and other post-graduate students in the field of suicide prevention; and iv) to raise awareness of suicide and its prevention in the wider community.

\section{Operational budget}

The clinic operates on a cost-recovery basis, with Medicare client fees representing the most relevant source of funding. Services are provided through the Medicare system making all visits bulk-billed. This was made possible with the Better Outcomes in Mental Health Care Program, implemented in Australia in 2003. One of the components of the program was the Better Access initiative, which aimed to improve community access to mental health professionals by enabling general practitioners (GPs) to refer their patients to allied health professionals (e.g., psychiatrists, clinical psychologist, registered psychologists). Through Better Access, patients can receive up to 10 (14 in exceptional cases) clinical interventions individual and/or group therapy sessions within a calendar year. The mental health nurse is supported by the national Mental Health Nurse Incentive Program.

\section{Referral pathways}

Patients are in most cases referred to the clinic following a presentation to ED, or discharge from mental health inpatient facilities in South East Queensland. Patients are also referred by GPs, and in some case their family or friends.

\section{Client selection criteria and consent procedures}

Patients are always informed about the research nature and operation of the clinic, as well as available treatment options provided. This is in agreement with Taylor et al. (2009, data not cited), who found that especially suicidal patients are generally provided with insufficient information about their care.

A few selection criteria apply for acceptance to treatment at the LPC. These include: age of 18 years and over, and current suicidal ideation or recent attempt(s). Exclusion criteria concern imminent risk of suicide, acute psychosis, and severe alcohol/substance abuse disorders.

Clients consent in writings to treatment and research participation. The Ethics Committee of Griffith University approved the functioning of the LPC.

\section{Treatment}

The clinic offers a variety of psychotherapeutic approaches depending on clinical needs, including individual and group psychotherapeutic treatment (based on dialectical behavior therapy, cognitive behavioral therapy, mindfulness, psychodynamic psychotherapy), nearly always supplemented by pharmacotherapy. The aim of the LPC is to develop innovative approaches of clinical practice to counteract suicidal behaviors. Currently, evaluation of one such treatment is in place at the clinic (Emotion Modulation Therapy). Particular care is taken in creating a nurturing and sup- portive environment for clients in order to facilitate the treatment process.

\section{Follow-up}

Patients are contacted at 6,12 , and 24 months after the end of their treatment. Each patient consents to this agenda of contacts at the beginning of treatment. Follow-ups include a phone call by a clinician (not the treating one) treating the patient), and involves assessment of health and well being, including possible re-occurrence of suicidality. A battery of tests (described below) is sent to patients to complete and return in a reply-free envelope.

An intention to treat approach inspires the follow-ups, which are primarily planned to guarantee continuity of attention/care to clinic clients. Intention to treat designs permit collection of data on the non-response and dropout figures, which are useful in evaluating outcomes. ${ }^{10}$

\section{Life Promotion Clinic patients}

From September 2008 (when the clinic was equipped as described above) to August 2011, 63 men and 175 women attended the LPC (patients that did not commence treatment are excluded from these figures). Their mean age was 33.6 years; 35.6 years (range 19-61 years)

Table 1. Demographic characteristics of patients, by gender.

\begin{tabular}{|c|c|c|c|c|c|c|}
\hline \multirow[t]{2}{*}{ Characteristic } & \multicolumn{2}{|c|}{ Males } & \multicolumn{2}{|c|}{ Females } & \multicolumn{2}{|c|}{ Total } \\
\hline & $\mathbf{N}$ & $\%$ & $\mathbf{N}$ & $\%$ & $\mathbf{N}$ & $\%$ \\
\hline \multicolumn{7}{|l|}{ Aboriginal and Torres Strait Islander status* } \\
\hline Aboriginal and Torres Strait Islander & 3 & 4.9 & 10 & 5.9 & 13 & 5.6 \\
\hline Other Australian & 58 & 95.1 & 160 & 94.1 & 218 & 94.4 \\
\hline \multicolumn{7}{|l|}{ Country of birth** } \\
\hline Australia & 46 & 73.0 & 145 & 82.9 & 191 & 80.3 \\
\hline Other country & 17 & 27.0 & 30 & 17.1 & 47 & 19.7 \\
\hline \multicolumn{7}{|l|}{ State*** } \\
\hline Queensland & 61 & 96.8 & 172 & 98.3 & 233 & 97.9 \\
\hline Other state & 2 & 3.2 & 3 & 1.7 & 5 & 2.1 \\
\hline \multicolumn{7}{|l|}{ Living arrangement ${ }^{\circ}$} \\
\hline With friend/other relative & 27 & 44.3 & 79 & 45.7 & 106 & 45.3 \\
\hline Alone & 15 & 24.6 & 34 & 19.7 & 49 & 20.9 \\
\hline With spouse/partner/children & 14 & 23.0 & 39 & 22.5 & 53 & 22.6 \\
\hline Other shared/institutional & 5 & 8.2 & 21 & 12.1 & 26 & 11.1 \\
\hline \multicolumn{7}{|l|}{ Marital status ${ }^{\circ \circ}$} \\
\hline Single & 40 & 64.5 & 103 & 59.2 & 143 & 60.6 \\
\hline Married/De facto & 15 & 24.2 & 39 & 22.4 & 54 & 22.9 \\
\hline Divorced/Separated/Widowed & 7 & 11.0 & 32 & 18.0 & 39 & 17.0 \\
\hline \multicolumn{7}{|l|}{ Education level ${ }^{\circ \circ \circ}$} \\
\hline Post-school (Under/Postgraduate) & 16 & 25.8 & 42 & 24.6 & 58 & 24.9 \\
\hline TFE/Trade & 8 & 12.9 & 23 & 13.5 & 31 & 13.3 \\
\hline Year 12 or less & 38 & 61.3 & 106 & 62.0 & 144 & 61.8 \\
\hline \multicolumn{7}{|l|}{ Employment status\# } \\
\hline Employed (any modality) & 21 & 34.4 & 55 & 31.6 & 76 & 32.3 \\
\hline Unemployed receiving pension/benefit & 11 & 18.0 & 37 & 21.3 & 48 & 20.4 \\
\hline Unemployed & 25 & 41.0 & 60 & 34.5 & 85 & 36.2 \\
\hline Out of the labor force (student/retired) & 4 & 6.6 & 22 & 12.6 & 26 & 11.1 \\
\hline
\end{tabular}

*Fisher exact test $=1.000$, missing $=7(2.9 \%){ }^{* *} \chi^{2}=2.83, \mathrm{df}=1, \mathrm{P}=0.092 .{ }^{* * *}$ Fisher exact test $=0.610{ }^{\circ} \chi^{2}=1.54, \mathrm{df}=3, \mathrm{P}=0.673$, missing $=4(1.7 \%)$. ${ }^{\circ} \chi^{2}=1.67, \mathrm{df}=2, \mathrm{P}=0.434$, missing $=2(0.8 \%) .{ }^{\circ \circ} \chi^{2}=0.04, \mathrm{df}=2, \mathrm{P}=0.979$, missing $=5$ (2.1\%). $\chi^{2}=2.38, \mathrm{df}=3, \mathrm{P}=0.497$, missing $=3(1.3 \%)$ 
for males and 32.8 years (17-65 years) for females $(t=1.68, \mathrm{NS})$.

\section{Assessments}

Routinely, the first appointment lasts approximately two hours. As said, patients are aware that LPC is a research clinic. Before meeting with a clinician (a consultant psychiatrist or a psychiatry registrar), patients are requested to sign consent form, and to fill in a number of self-report questionnaires. These include: i) the Beck Scale for Suicide Ideation (BSS);11 ii) the Beck Hopelessness Scale (BHS);12 iii) the Depression, Anxiety and Stress Scales (DASS); 13 and, iv) the Impulsiveness Questionnaire (IVE). 14

\section{Demographic profile of clients}

During the three years considered, 238 patients attended the LPC; most of them (58.4\%) were 15-34 years old. Main demographic characteristics are presented in Table 1. There were no significant differences between male and female patients in terms of Aboriginal and Torres Strait Islander status, country of birth, living arrangements, marital and employment status, and education.

\section{Referral and clinical profile of clients}

Of the 238 patients, 127 (53.4\%) were referred by a hospital-based mental health professional; 91 (38.2\%) by a GP. Small numbers of patients were referred by a non-governmental organization $(n=11 ; 4.6 \%)$, or a family member or friend who attended the clinic previously $(\mathrm{n}=9 ; 3.8 \%)$.

All diagnoses were formulated by the aid of the Structured Clinical Interview for DSM Disorders (Out-Patient Version). ${ }^{15}$ Most patients presented with co-morbidities: $43.7 \%$ ( $n=104)$ had two psychiatric diagnoses; $12.2 \%$ $(\mathrm{n}=29)$ had three; and 3.8\% $(\mathrm{n}=9)$ had four diagnoses, while $40.3 \%(n=96)$ had one diagnosis only. There were no significant differences in the number of diagnoses by gender $(\mathrm{t}=0.18, \mathrm{NS})$. Uni-polar depression was the most frequent diagnosis in both genders, followed by anxiety and personality disorders (Table 2). Personality disorders were significantly more frequent in female patients, while psychotic disorders being prevalent in males. The majority of patients $(n=203,85.3 \%)$ were prescribed medications, in more than twothirds of cases antidepressants (Table 2). There were no significant differences by gender. More than three-quarters $(78.7 \%)$ of patients reported they had self-harmed at least once in their life, with intention to die during the most recent attempt (Table 3). Male patients appeared significantly more determined to kill themselves than females (Table 4). The average BSS score of suicide ideators was $20.0(\mathrm{SD}=7.1)$, close to detect a significant difference between sexes $(\mathrm{t}=1.98, \mathrm{P}=0.051)$. At the time of their first appointment, the majority of patients (62.2\%) reported a moderate level of hopelessness, with an additional $17.0 \%$ presenting high levels. The mean BHS score was 11.1 ( $\mathrm{SD}=3.5)$, with no significant differences between males $(\mathrm{t}=0.22, \mathrm{NS})$.
According to DASS scores, the majority of the patients reported severe level of depression $(n=158,78.6 \%)$ and anxiety $(n=144$, $72.7 \%$ ), while 128 patients (64.3\%) reported a severe level of stress (Figure 1). There were no significant differences between male and female patients in the average scores of depression ( $\mathrm{t}=0.73, \mathrm{NS})$, anxiety $(\mathrm{t}=1.55$, NS) or stress $(\mathrm{t}=1.22, \mathrm{NS})$.

The patients of the LPC were found to have relatively high impulsiveness scores $(9.9$, $\mathrm{SD}=4.0$ ), with no significant differences between male and female patients ( $\mathrm{t}=0.87, \mathrm{NS})$.

\section{Discussion and Conclusions}

A few limitations should first be acknowledged. People who seek specialized help from the LPC might not represent the general population of suicidal individuals. Furthermore, given the research nature of the clinic, a number of patients whose symptoms affect mental concentration may have found it difficult to attend the LPC, due to the extent of the protocol required for initial assessment. The effective management of the clinic has been affected by insufficient number of staff, which is still reflected in the limited operating time for clients (i.e., two days per week). The increasing number of referrals shows the need for an expansion of services. Given the characteristics of the clientele, it would be inappropriate to impose waiting time beyond two weeks from referral. The LPC is a public outpatient service

Table 2. Psychiatric diagnoses and prescribed medications of patients (multiple diagnoses and medications allowed).

\begin{tabular}{|c|c|c|c|c|c|c|c|c|c|}
\hline & \multicolumn{2}{|c|}{ Males } & \multicolumn{2}{|c|}{ Females } & \multicolumn{2}{|c|}{ Total } & \multirow[t]{2}{*}{$\chi^{2}(\mathrm{df}=1)$} & \multirow[t]{2}{*}{$\mathbf{P}$} & \multirow[t]{2}{*}{$\mathbf{F}$} \\
\hline & $\mathbf{N}$ & $\%$ & $\mathbf{N}$ & $\%$ & $\mathbf{N}$ & $\%$ & & & \\
\hline \multicolumn{10}{|c|}{ Psychiatric diagnoses* } \\
\hline Unipolar depression & 43 & 68.2 & 112 & 64.0 & 155 & 65.1 & 0.37 & 0.543 & \\
\hline Anxiety (incl PTSD and trauma) & 25 & 39.7 & 73 & 41.7 & 98 & 41.2 & 0.08 & 0.779 & \\
\hline Personality disorder & 17 & 27.0 & 72 & 41.1 & 89 & 37.4 & 3.97 & 0.046 & \\
\hline Substance use disorder & 11 & 17.4 & 20 & 11.4 & 31 & 13.0 & 1.49 & 0.223 & \\
\hline Bipolar depression & 5 & 7.9 & 11 & 6.3 & 16 & 6.7 & 0.20 & 0.654 & \\
\hline Psychotic disorder & 6 & 9.5 & 3 & 1.7 & 9 & 3.8 & - & - & 0.012 \\
\hline Other or vague disorder & 2 & 3.2 & 6 & 3.4 & 8 & 3.4 & - & - & 1.000 \\
\hline Eating disorder & 1 & 1.6 & 6 & 3.4 & 7 & 2.9 & - & - & 0.679 \\
\hline Developmental disorder & 0 & - & 1 & 0.6 & 1 & 0.4 & - & - & 1.000 \\
\hline \multicolumn{10}{|c|}{ Prescribed medications } \\
\hline Any prescribed medications & 56 & 88.8 & 147 & 84.0 & 203 & 85.3 & 0.88 & 0.347 & \\
\hline Antidepressant & 50 & 79.4 & 136 & 77.7 & 186 & 78.2 & 0.74 & 0.786 & \\
\hline Sedative and/or anxiolytic & 23 & 36.5 & 50 & 28.6 & 73 & 30.7 & 1.37 & 0.241 & \\
\hline Antipsychotic & 18 & 28.6 & 41 & 23.4 & 59 & 24.8 & 0.66 & 0.418 & \\
\hline Other mood stabilizer & 6 & 9.5 & 22 & 12.6 & 28 & 11.8 & 0.41 & 0.520 & \\
\hline Lithium & 2 & 3.2 & 8 & 4.6 & 10 & 4.2 & - & - & 1.000 \\
\hline
\end{tabular}

*All the patients had at least one psychiatric diagnosis. PTSD, Post Traumatic Stress Disorder; P, P-value; F, Fisher's ex test. 
Table 3. Past suicide attempts and intention to die during the most recent attempt, by gender.

\begin{tabular}{lcccccc} 
& \multicolumn{3}{c}{ Males } & Females & Total \\
& N & $\%$ & N & $\%$ & N & $\%$ \\
Never & 15 & 27.3 & 29 & 19.1 & 44 & 21.3 \\
Once & 12 & 21.8 & 27 & 17.8 & 39 & 18.8 \\
\hline Two or more times & 28 & 50.9 & 96 & 63.2 & 124 & 59.9 \\
& Wish to die during past suicide attemptb & & \\
Low & 2 & 5.0 & 7 & 5.7 & 9 & 5.6 \\
Moderate & 6 & 15.0 & 34 & 27.9 & 40 & 24.7 \\
\hline High & 32 & 80.0 & 81 & 66.4 & 113 & 69.8 \\
Not applicable* & 15 & 23.8 & 29 & 16.6 & 44 & 18.5 \\
\hline
\end{tabular}

${ }^{a} \chi^{2}=2.64$, df $=2, P=0.267$, missing $=31(13 \%)$. ${ }^{b} \chi^{2}=2.76, d f=2, P=0.252$, missing $=32$ (13.5\%). * Patients with no prior suicide attempt.

Table 4. Active and passive suicidal ideation (items 4 and 5 of the BSS), by gender.

\begin{tabular}{|c|c|c|c|c|c|c|}
\hline & \multicolumn{2}{|c|}{ Males } & \multicolumn{2}{|c|}{ Females } & \multicolumn{2}{|c|}{ Total } \\
\hline & $\mathbf{N}$ & $\%$ & $\mathbf{N}$ & $\%$ & $\mathbf{N}$ & $\%$ \\
\hline \multicolumn{7}{|c|}{ Passive desire to kill oneself* } \\
\hline No desire & 22 & 40.7 & 52 & 34.9 & 74 & 36.5 \\
\hline Weak desire & 18 & 33.3 & 69 & 46.3 & 87 & 42.9 \\
\hline Moderate desire & 14 & 25.9 & 28 & 18.8 & 42 & 20.7 \\
\hline \multicolumn{7}{|c|}{ Active desire to kill oneself** } \\
\hline No desire & 13 & 24.1 & 49 & 32.0 & 62 & 30.0 \\
\hline Weak desire & 19 & 35.2 & 69 & 45.1 & 88 & 42.5 \\
\hline Moderate desire & 22 & 40.7 & 35 & 22.9 & 57 & 27.5 \\
\hline
\end{tabular}

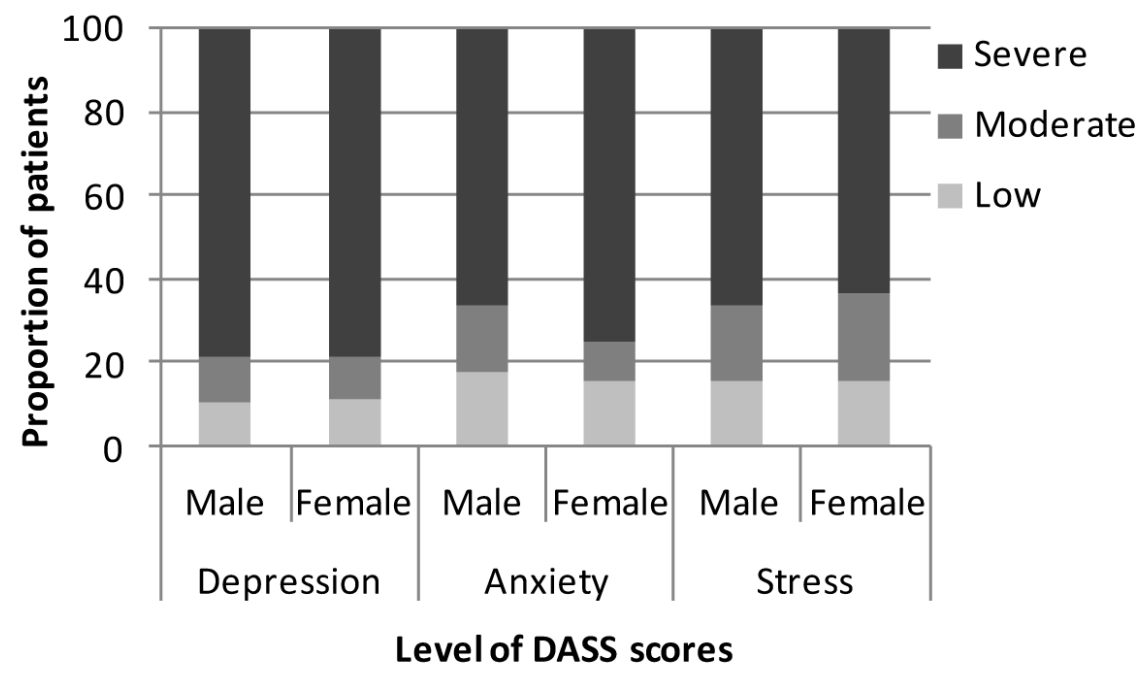

Figure 1. Patients by levels of depression, anxiety and stress. Missing: Depression: 37 (15.5\%), Anxiety: 40 (16.8\%), Stress: 39 (16.4\%) dedicated to the care of suicide ideators and attempters. As such, it represents a rare opportunity for studying a population of subjects that can be particularly challenging in terms of clinical management and treatment. And, in fact, patients that attend the LPC are highly suicidal and in great need for help. Most of them report at least one self-harming episode during lifetime, accompanied by persistent suicide ideation. In patients seen so far, males presented significantly more often active desire to kill themselves and higher level of suicidal ideation than females. For psychiatry registrars, having their training at the LPC represents an important opportunity for professional growth, especially knowing that - particularly in private settings - mental health professionals are reluctant to treat suicidal individuals, and chances to gain clinical experience are thus limited.16,17

Our finding that the majority of patients were single, had 12 years or less of education, and were mostly unemployed (or receiving disability support pension or other benefits) contradicts the results of a WHO Mental Health Survey, ${ }^{2}$ showing that receiving treatment after engaging in suicidal behavior was predicted by being married, having higher education and income. This seems to suggest that bulk billing of services may be an important aspect in the care of suicidal individuals, making treatment accessible to all social categories.

\section{References}

1. Milner A, De Leo D. Who seeks treatment where? Suicidal behaviors and health care: evidence from a community survey. $\mathrm{J}$ Nerv Ment Dis 2010;198:412-9.

2. Bruffaerts R, Demyttenaere K, Hwang I, et al. Treatment of suicidal people around the world. Br J Psychiatry 2011;199:64-70.

3. Taylor TL, Hawton K, Fortune S, Kapur N. Attitudes towards clinical services among people who self-harm: systematic review. Br J Psychiatry 2009;194:104-10.

4. Lizardi D, Stanley B. Treatment engagement: a neglected aspect in the psychiatric care of suicidal patients. Psychiatr Serv 2010;61:1183-91.

5. Meehan J, Kapur N, Hunt IM, et al. Suicide in mental health in-patients and within 3 months of discharge: national clinical survey. Br J Psychiatry 2006;188:129-34.

6. Saunders KEA, Hawton K, Fortune S, Farrell S. Attitudes and knowledge of clinical staff regarding people who self-harm: a systematic review. J Affect Disord 2012;139: 205-16.

7. Beghi M, Rosenbaum JF. Risk factors for fatal and nonfatal repetition of suicide 
attempt: a critical appraisal. Curr Opin Psychiatry 2010;23:349-55.

8. Owens D, Horrocks J, House, A. Fatal and non-fatal repetition of self-harm. systematic review. Br J Psychiatry 2002;181:1939.

9. De Leo D, Cerin E, Spathonis K, Burgis S. Lifetime risk of suicide ideation and attempts in an Australian community: prevalence, suicidal process, and helpseeking behaviour. J Affect Disord 2005; 86:215-24.

10. Schottenbauer MA, Glass CR, Arnkoff DB, et al. Nonresponse and dropout rates in outcome studies on PTSD: review and methodological considerations. Psychiatry 2008;71:134-68.

11. Beck AT, Steer RA. Beck hopelessness scale. San Antonio, TX: Psychological Corporation; 1993.

12. Beck AT, Steer RA. Manual for the Beck scale for suicide ideation. San Antonio, TX: Psychological Corporation; 1993.

13. Lovibond SH, Lovibond PF. Manual for the depression anxiety stress scales. 2nd ed. Sydney: Psychology Foundation; 1995.

14. Eysenck HJS, Eysenck BJ. Eysenck personality scales (EPS Adult). London: Hodder \&
Stoughton; 1991.

15. Spitzer RL, Gibbon M, Williams J. Structured clinical interview for Axis I DSM-IV disorders. New York: New York State Psychiatric Institute: Biometrics Research, 1994.

16. De Gioannis A, De Leo D. Managing suicidal patients in clinical practice. Open $\mathrm{J}$ Psychiatry 2012;2:49-60.

17. Kaye NS, Soreff SM. The psychiatrist's role, responses, and responsibilities when a patient commits suicide. Am J Psychiatry 1991;148:739-43. 